\title{
Consistency of Generalized Maximum Spacing Estimates
}

\author{
Magnus Ekström \\ Department of Mathematical Statistics \\ Umeå University \\ S-901 87 Umeå, Sweden
}

\begin{abstract}
General methods for the estimation of distributions can be derived from approximations of certain information measures. For example, both the maximum likelihood (ML) method and the maximum spacing (MSP) method can be obtained from approximations of the Kullback-Leibler information. The ideas behind the MSP method, whereby an estimation method for continuous univariate distributions is obtained from an approximation based on spacings of an information measure, were used by Ranneby and Ekström (1997) (using simple spacings) and Ekström (1997) (using high order spacings) to obtain a class of estimation methods, called generalized maximum spacing (GMSP) methods. In the present paper, GMSP methods will be shown to give consistent estimates under general conditions, comparable to those of Bahadur (1971) for the ML method, and those of Shao and Hahn (1996) for the MSP method. In particular, it will be shown that GMSP methods give $L^{1}$ consistent estimates in any family of distributions with unimodal densities, without any further conditions on the distributions.
\end{abstract}

Key words and phrases: Estimation, Spacings, Consistency, Maximum spacing method, Unimodal density

1991 AMS subject classification: $62 \mathrm{~F} 12,62 \mathrm{G} 20,60 \mathrm{~F} 15$ 



\section{Introduction}

The most frequently used general estimation method is that of maximum likelihood (ML). In Cheng and Amin (1983), and independently in Ranneby (1984), an alternative to the ML method, the maximum spacing (MSP) method, is introduced to estimate finite dimensional parameters in continuous univariate distributions. Ranneby based the method on an approximation of the Kullback-Leibler information, using simple spacings. The MSP-method is appealing since it gives estimators that have asymptotic properties closely parallel to ML-estimators. Moreover the MSP-method works also in situations where the ML-method breaks down, e.g. for three parameter Weibull and gamma distributions, mixtures of continuous distributions and "heavy-tailed" continuous distributions.

Ranneby (1984) raises the question as to whether it is possible to obtain better methods by approximations of information measures other than the KullbackLeibler information, such as the Hellinger distance. In Ranneby and Ekström (1997) a class of estimation methods, called generalized maximum spacing (GMSP) methods, is derived from approximations based on simple spacings of so called $\phi-$ divergences (introduced by Csiszár (1963)). See also Ghosh and Jammalamadaka (1996) for closely related ideas. Note that information measures such as the Kullback-Leibler information, the Jeffreys divergence and the Hellinger distance, all are $\phi$-divergences or functions of a $\phi$-divergence. Under particular regularity conditions Ghosh and Jammalamadaka (1996) show that GMSP-estimates (based on simple spacings) are asymptotically normal and that the lower bound in the Cramér-Rao inequality is reached only for the GMSP-method obtained from the approximation of the Kullback-Leibler information, i.e. the MSP-method. On the other hand, when the regularity conditions do not hold, e.g. the three parameter Weibull model, Ghosh and Jammalamadaka show in a simulation study that GMSP-methods other than the MSP-method can perform better in terms of mean square error. Moreover, the performance of GMSP-estimators can be improved by the use of high order spacings, see Ekström (1997).

In this paper strong consistency theorems will be given for GMSP-estimators under general conditions comparable to those of Bahadur (1971) for ML-estimators and of Shao and Hahn (1996) for MSP-estimators. In section 2 results will be given for GMSP-estimators by the use of simple spacings. These results include a theorem for families of distributions with unimodal densities, where the estimator of the underlying unimodal density will be shown to be $L^{1}$ consistent, without any further conditions on the model. In section 3, estimators based on approximations of the Kullback-Leibler information using high order spacings are considered. Such estimators, using spacings of fixed order, have previously been studied by Roeder (1990) and Ekström (1997b). Here we will look at the case when the order is allowed to increase to infinity with the sample size. 


\section{Consistency results for GMSP-estimates us- ing simple spacings}

For each probability measure $P$, from a given family $\mathcal{P}$ of probability measures dominated by the Lebesgue measure $\mu$, the density and distribution functions will be denoted $f_{P}$ and $F_{P}$, respectively.

Suppose that $\xi_{1}, \ldots, \xi_{n}$ is an i.i.d. sample from $P_{0} \in \mathcal{P}$ and that the aim is to estimate the unknown $P_{0}$. Let $-\infty=\xi_{(0)} \leq \xi_{(1)} \leq \ldots \leq \xi_{(n)} \leq \xi_{(n+1)}=\infty$ denote the ordered values.

The GMSP-estimator of $P_{0}$, as defined in Ranneby and Ekström (1997), is any probability measure $P$ in $\mathcal{P}$ that maximizes

$$
S_{\Psi, n}(P)=\frac{1}{n+1} \sum_{j=0}^{n} \Psi\left((n+1)\left(F_{P}\left(\xi_{(j+1)}\right)-F_{P}\left(\xi_{(j)}\right)\right)\right),
$$

where $\Psi$ is some suitable concave function on $R^{+}$. This class of estimators are derived from approximations of Csiszár's $\phi$-divergences $(\Psi=-\phi)$, e.g. $\Psi(x)=\log x$ and $\Psi(x)=-|1-\sqrt{x}|^{2}$ give estimators based on the Kullback-Leibler information and the Hellinger distance respectively.

Example: Assume that $P_{0} \in \mathcal{P}$, the class of all probability measures dominated by $\mu$. Then, for any concave function $\Psi$, any probability measure $\tilde{P}_{\Psi, n} \in \mathcal{P}$, with a corresponding distribution function $\tilde{F}_{\Psi, n}$ satisfying $\tilde{F}_{\Psi, n}\left(\xi_{(i)}\right)=i /(n+1)$, $0 \leq i \leq n+1$, is a GMSP-estimate. Since $\sup _{x \in R}\left|\tilde{F}_{\Psi, n}(x)-F_{n}(x)\right| \leq 1 /(n+1)$, where $F_{n}$ is the empirical distribution function, it follows by the Glivenko-Cantelli Theorem that $\tilde{F}_{\Psi, n}$ converges weakly to the true underlying distribution $F_{P_{0}}$.

Since the GMSP-estimator does not necessarily exist $\operatorname{(sup}_{P \in \mathcal{P}} S_{\Psi, n}(P)$ is not necessarily attained for any $P \in \mathcal{P}$ ) we define the asymptotic GMSP-estimate $\hat{P}_{\bar{\Psi}, n}, \hat{P}_{\Psi, n} \in \mathcal{P}$ for all $n$, such that

$$
\varliminf_{n \rightarrow \infty} S_{\Psi, n}\left(\hat{P}_{\Psi, n}\right) \stackrel{\text { a.s. }}{\geq} \varlimsup_{n \rightarrow \infty} S_{\Psi, n}\left(P_{0}\right) .
$$

As the strong law of large numbers is a cornerstone in Wald's (1949) classical proof of strong consistency of the ML-estimator for the general case, the following two limit theorems play significant roles in the proof of strong consistency of the (asymptotic) GMSP-estimator.

For later references we state some assumptions used in these two limit theorems here:

Assumption $A_{1}$ : The true underlying density $f_{P_{0}}(\cdot)$ is right-continuous $\mu-$ almost everywhere.

Assumption $A_{2}$ : The function $\Psi(t), t \in R^{+}$, satisfies the following conditions: 
(i) $\Psi$ is strictly concave,

(ii) $\Psi^{+}(t) / t \rightarrow 0$ as $t \rightarrow \infty$, where $\Psi^{+}(t)=\max (0, \Psi(t))$,

(iii) $\Psi(t)$ is either equal to $\log t$ or is bounded from below.

Theorem 1 Let $\xi_{1}, \ldots, \xi_{n}$ be a sequence of i.i.d. random variables with density function $f_{P_{0}}$. Suppose that assumption $A_{2}$ is valid. Then

$$
\lim _{n \rightarrow \infty} S_{\Psi, n}\left(P_{0}\right) \stackrel{\text { a.s. }}{=} \int_{R} \Psi(x) e^{-x} d x \text {. }
$$

Theorem 2 Let $\xi_{1}, \ldots, \xi_{n}$ be a sequence of i.i.d. random variables with density function $f_{P_{0}}$. Suppose that assumptions $A_{1}, A_{2}(i)$ and $A_{2}(i i)$ are valid. Then, for any finite union of intervals $\mathcal{I}$ and any finite measure $Q$ dominated by the Lebesgue measure $\mu$, with $f_{Q}(x)=\frac{d Q}{d \mu}(x)$ and $F_{Q}(x)=\int_{-\infty}^{x} f_{Q}(x) d x$, almost surely,

$$
\varlimsup_{n \rightarrow \infty} \frac{1}{n+1} \sum_{j \in J_{\mathcal{I}}} \Psi\left((n+1)\left(F_{Q}\left(\xi_{(j+1)}\right)-F_{Q}\left(\xi_{(j)}\right)\right)\right) \leq \int_{\mathcal{I}} \int_{R^{+}} \Psi\left(y f_{Q}(x)\right) p_{*}(x, y) d y d x,
$$

where $J_{\mathcal{I}}=\left\{j:\left[\xi_{(j)}, \xi_{(j+1)}\right] \subseteq \mathcal{I}, 0 \leq j \leq n\right\}$ and $p_{*}(x, y)=f_{P_{0}}^{2}(x) e^{-y f_{P_{0}}(x)}, y>0$.

Let $\mathcal{S}$ be the space of all subprobability measures on the real line endowed with the topology of vague convergence. A sequence $\left\{Q_{n}\right\}$ in $S$ converges vaguely to $Q \in \mathcal{S}$, written $Q_{n} \stackrel{v}{\rightarrow} Q$, if for all continuous real valued functions $h$ on the real line with compact support, $\int_{-\infty}^{\infty} h(x) d Q_{n}(x) \rightarrow \int_{-\infty}^{\infty} h(x) d Q(x), n \rightarrow \infty$. With this definition of convergence, $\mathcal{S}$ becomes a metrizable, compact, topological space (see Bauer (1972)). Let $d$ be a distance function on $\mathcal{S} \times \mathcal{S}$ such that, for any sequence $\left\{Q_{n}\right\}$ in $\mathcal{S}$ and $Q \in \mathcal{S}, d\left(Q_{n}, Q\right) \rightarrow 0$, as $n \rightarrow \infty$, if and only if $Q_{n} \stackrel{\cup}{\rightarrow} Q$, as $n \rightarrow \infty$. Let $\overline{\mathcal{P}}$ be the closure of $\mathcal{P}$ in $\mathcal{S}$. This makes $\overline{\mathcal{P}}$ a compact space.

For any $Q \in \overline{\mathcal{P}}$ and any positive $r$, let

$$
g_{Q}(x, r)=\sup \left\{f_{P}(x): P \in \mathcal{P}, d(Q, P)<r\right\} .
$$

As in Bahadur (1971), $\overline{\mathcal{P}}$ will be called a "suitable compactification" of $\mathcal{P}$ if $g_{Q}(x, r), Q \in \overline{\mathcal{P}}$, is measurable for all $r>0$ and if $\gamma_{Q}(x)=\lim _{r \downarrow 0} g_{Q}(x, r)$ satisfies $\int_{R} \gamma_{Q}(x) d x \leq 1$. This implies that $\gamma_{Q}(x)=f_{Q}(x)$ a.e. for all $Q \in \mathcal{P}$. As noted by Bahadur, if $Q \in \overline{\mathcal{P}} \backslash \mathcal{P}$, then $\gamma_{Q}$ is not necessarily a version of $\frac{d Q}{d \mu}(x)$ and the subprobability $Q$ may not even be dominated by $\mu$.

Consistency of $\hat{P}_{\Psi, n}$ will be proved under assumptions $A_{1}, A_{2}$ and the following additional three assumptions: 
Assumption $A_{3}: \overline{\mathcal{P}}$ is a "suitable compactification" of $\mathcal{P}$.

As noted by Bahadur (1971), assumption $A_{3}$ is independent of the metric $d$, i.e. it holds with some choice of $d$ if and only if it holds for every choice.

Assumption $A_{4}$ : For each $Q \in \overline{\mathcal{P}} \backslash \mathcal{P}, \mu\left\{x: \gamma_{Q}(x) \neq f_{P_{0}}(x)\right\}>0$.

Assumption $A_{5}$ : For any $Q \in \overline{\mathcal{P}}$,

$$
\lim _{M \rightarrow \infty} \lim _{r \downarrow 0} P_{0}\left(\overline{\left\{x: g_{Q}(x, r) \geq \bar{M}\right\}}\right)=0 .
$$

For a discussion on assumptions $A_{3}-A_{5}$, in comparison with classical regularity conditions for consistency of ML-estimators, see Shao and Hahn (1996). The following three theorems generalize results of Shao and Hahn (1996), who considered the special case $\Psi(t)=\log t$.

Theorem 3 Let $\xi_{1}, \ldots, \xi_{n}$ be i.i.d. random variables according to $P_{0}$ in $\mathcal{P}$, a class of probability measures on $R$ dominated by the Lebesgue measure. Then, under assumptions $A_{1}-A_{5}$, any sequence $\left\{\hat{P}_{\Psi, n}\right\}$ is consistent, that is $d\left(\hat{P}_{\Psi, n}, P_{0}\right) \stackrel{\text { a.a. }}{\rightarrow} 0$ as $n \rightarrow \infty$.

Theorem 4 Under the assumptions of Theorem 3 , if $\overline{\mathcal{P}} \backslash \mathcal{P}$ is a closed set, then with probability 1 a GMSP-estimator exists for all sufficiently large $n$, and any GMSP-estimator sequence is consistent.

Proof. The proof for the special case $\Psi(t)=\log t$ is given in Shao and Hahn (1996). The proof for the general case is almost identical (a slight change of notation is needed and the reference to Theorem 3.1 in Shao and Hahn (1996) should be changed to Theorem 3 above).

Theorem 5 Let $\xi_{1}, \ldots, \xi_{n}$ be i.i.d. random variables with density $f_{P_{0}}, P_{0} \in \mathcal{P}$, where $\mathcal{P}$ is a family of probability measures with unimodal densities. Then, under assumption $A_{2}$, any asymptotic GMSP-estimator is consistent for $f_{P_{0}}$ in $L^{1}$ distance, i.e.

$$
\int_{R}\left|f_{\hat{P}_{\psi, n}}(x)-f_{P_{0}}(x)\right| d x \stackrel{a . s .}{\rightarrow} 0, \text { as } n \rightarrow \infty \text {. }
$$

\section{Consistency results for GMSP-estimates us- ing high order spacings, where the order of the spacings is allowed to increase to infinity}

The notation of the previous section will be used unless otherwise stated. The GMSP-estimator of $P_{0}$, as defined in Ekström (1997b), will be considered, i.e. 
any probability measure $P \in \mathcal{P}$ that maximizes

$$
\frac{1}{n} \sum_{j=0}^{n-m+1} \Psi\left(\frac{n+1}{m}\left(F_{P}\left(\xi_{(j+m)}\right)-F_{P}\left(\xi_{(j)}\right)\right)\right),
$$

where $m$ is a positive integer that may depend on $n$, and where $\Psi$ is some suitable concave function. By the use of high order spacings, "better" approximations of Csiszár's $\phi$-divergences can be obtained, indeed in Ekström (1997b) it was found that for many choices of $\Psi$, high order spacings give better estimators, e.g. in terms of robustness and lower variance. Previously only fixed values of $m$ have been considered, but here we will look at the case where the order $m$ of the spacings is allowed to increase to infinity with the sample size. However we will restrict attention to the particular case $\Psi(x)=\log x$, i.e. to GMSP-estimators based on approximations of the Kullback-Leibler information. In Roeder (1990) secondorder spacings were recommended (when $\Psi(x)=\log x$ ). She found through simulations that the second-order spacings are more robust to near ties, as expected, and that the estimators based on second-order spacings performed as well as those based on simple spacings. No advantage to letting the order of spacings tend to infinity when $\Psi(x)=\log x$ is presently known, so this section is merely of theoretical interest.

Define, for $P \in \mathcal{P}$,

$$
S_{n}^{(m)}(P)=\frac{1}{n} \sum_{j=0}^{n-m+1} \log \left(\frac{n+1}{m}\left(F_{P}\left(\xi_{(j+m)}\right)-F_{P}\left(\xi_{(j)}\right)\right)\right) .
$$

Since the GMSP-estimate does not necessarily exist, we define the asymptotic GMSP-estimator $\hat{P}_{n}^{\left(m_{n}\right)}, \hat{P}_{n}^{\left(m_{n}\right)} \in \mathcal{P}$ for all $n$, satisfying

$$
\begin{aligned}
\varliminf_{n \rightarrow \infty} S_{n}^{\left(m_{n}\right)}\left(\hat{P}_{n}^{\left(m_{n}\right)}\right) & \stackrel{a . s .}{\geq} \lim _{n \rightarrow \infty} S_{n}^{\left(m_{n}\right)}\left(P_{0}\right) \\
& \stackrel{\text { a.s. }}{=} \lim _{n \rightarrow \infty}\left(\psi\left(m_{n}\right)-\log m_{n}\right),
\end{aligned}
$$

where $\psi(x)=\frac{d}{d x} \log \Gamma(x)$ is the digamma function and where $\left\{m_{n}\right\}$ is a nondecreasing sequence of positive integers such that $m_{n}=o(n)$. The equality in (2) follows from an application of Theorem 1 in Ekström (1997a). We add one remark to guard against misapprehension. To say $m_{n}=o(s(n))$, where $s(n) \rightarrow \infty$ as $n \rightarrow \infty$, does not exclude that $m_{n}$ can be a bounded sequence.

In order to prove general consistency of $\hat{P}_{n}^{\left(m_{n}\right)}$ we need the next one-sided strong limit theorem for logarithms of high order spacings.

Theorem 6 Let $\xi_{1}, \ldots \xi_{n}$ be a sequence of i.i.d. random variables with density function $f_{P_{0}}$, and let $\left\{m_{n}\right\}$ be a nondecreasing sequence of positive integers such that $m_{n}=o(n / \log n)$. Then, for any finite union of intervals $\mathcal{I}$ and any $f-$ nite measure $Q$ dominated by the Lebesgue measure $\mu$, with $f_{Q}(x)=\frac{d Q}{d \mu}(x)$ and 
$F_{Q}(x)=\int_{-\infty}^{x} f_{Q}(x) d x$, almost surely,

$$
\varlimsup_{n \rightarrow \infty} \frac{1}{n} \sum_{j \in J_{I}} \log \left(\frac{F_{Q}\left(\xi_{\left(j+m_{n}\right)}\right)-F_{Q}\left(\xi_{(j)}\right)}{F_{P_{0}}\left(\xi_{\left(j+m_{n}\right)}\right)-F_{P_{0}}\left(\xi_{(j)}\right)}\right) \leq \int_{\mathcal{I}} \log \left(\frac{f_{Q}(x)}{f_{P_{0}}(x)}\right) d F_{P_{0}}(x),
$$

where $J_{\mathcal{I}}=\left\{j:\left[\xi_{(j)}, \xi_{\left(j+m_{n}\right)}\right] \subseteq \mathcal{I}, 0 \leq j \leq n-m_{n}+1\right\}$.

Proof. See Corollary 3 in Ekström (1997a).

Theorem 7 Let $\xi_{1}, \ldots, \xi_{n}$ be i.i.d. random variables according to $P_{0}$ in $\mathcal{P}$, a class of probability measures on $R$ dominated by the Lebesgue measure, and let $\left\{m_{n}\right\}$ be a nondecreasing sequence of positive integers such that $m_{n}=o(n / \log n)$. Then, under assumptions $A_{3}-A_{5}$, any sequence $\left\{\hat{P}_{n}^{\left(m_{n}\right)}\right\}$ is consistent, that is $d\left(\hat{P}_{n}^{\left(m_{n}\right)}, P_{0}\right) \stackrel{\text { a.f. }}{\rightarrow} 0$ as $n \rightarrow \infty$.

As in the previous section it can be shown, under the conditions in the theorem above, and if in addition $\overline{\mathcal{P}} \backslash \mathcal{P}$ is a closed set, that a GMSP-estimator (i.e. a probability measure in $\mathcal{P}$ that maximizes (1) with $m=m_{n}$ ) exists for all sufficiently large $n$, and that any GMSP-estimator is consistent. Moreover, as a parallel to Theorem 5, it can be shown that $\hat{P}_{n}^{\left(m_{n}\right)}$ is $L^{1}$-consistent for any unimodal distribution without any extra conditions.

\section{Proofs}

\subsection{Proofs of results presented in section 2}

Before proving the first theorem some new notation will be introduced and two useful lemmas will be given. For each $i=1,2, \ldots, n$, define,

$$
\begin{aligned}
\eta_{i}(n)= & (n+1) \cdot \text { "the distance from } \xi_{i} \text { to the nearest observation to the } \\
& \text { right of } \left.\xi_{i} \text { " (this distance is defined as }+\infty \text { if } \xi_{i}=\max _{1 \leq j \leq n} \xi_{j}\right)
\end{aligned}
$$

and, for each finite measure $Q$ dominated by $\mu$, with $f_{Q}(x)=\frac{d Q}{d \mu}(x)$ and $F_{Q}(x)=$ $\int_{-\infty}^{x} f_{Q}(x) d x$,

$$
z_{i}(n, Q)=(n+1)\left(F_{Q}\left(\xi_{i}+\frac{\eta_{i}(n)}{n+1}\right)-F_{Q}\left(\xi_{i}\right)\right) .
$$

Then, for $P \in \mathcal{P}$,

$$
\begin{aligned}
S_{\Psi, n}(P) & =\frac{1}{n+1} \sum_{j=0}^{n} \Psi\left((n+1)\left(F_{P}\left(\xi_{(j+1)}\right)-F_{P}\left(\xi_{(j)}\right)\right)\right) \\
& =\frac{1}{n+1} \sum_{i=1}^{n} \Psi\left(z_{i}(n, P)\right)+\frac{1}{n+1} \Psi\left((n+1) F_{P}\left(\min _{1 \leq j \leq n} \xi_{j}\right)\right)
\end{aligned}
$$


Let $P_{* n}$ denote the joint probability distribution of $\left(\xi_{1}, \eta_{1}(n)\right)$. By a conditioning on $\xi_{1}=s$ it is easily seen that

$$
\begin{aligned}
& P_{* n}(x, y)=P_{* n}\left(\xi_{1} \leq x, \eta_{1}(n) \leq y\right) \\
& \quad= \begin{cases}\int_{-\infty}^{x}\left[1-\left(1-\left(F_{P_{0}}\left(s+\frac{y}{n+1}\right)-F_{P_{0}}(s)\right)\right)^{n-1}\right] f_{P_{0}}(s) d s & \text { if } 0<y<\infty \\
\int_{-\infty}^{x} f_{P_{0}}(s) d s & \text { if } y=\infty .\end{cases}
\end{aligned}
$$

The density function $p_{* n}(x, y)$ of $P_{* n}(x, y)$ is given by

$$
\begin{cases}\frac{n-1}{n+1} f_{P_{0}}(x) f_{P_{0}}\left(x+\frac{y}{n+1}\right)\left(1-\left(F_{P_{0}}\left(x+\frac{y}{n+1}\right)-F_{P_{0}}(x)\right)\right)^{n-2} & \text { if } 0<y<\infty \\ f_{P_{0}}(x) F_{P_{0}}(x)^{n-1} & \text { if } y=\infty\end{cases}
$$

with respect to the measure $\lambda$ which is the two-dimensional Lebesgue measure $\mu \times \nu$ on $R \times R^{+}$, and the Lebesgue measure $\mu$ on $R \times\{+\infty\}$.

In Ekström (1994) it is shown (under the assumption that $f_{P_{0}}(x)$ is rightcontinuous $\mu$-almost everywhere) that $P_{* n}$ converges weakly to $P_{*}$, where

$$
P_{*}(x, y)=\int_{-\infty}^{x}\left(1-e^{-y f_{P_{0}}(u)}\right) f_{P_{0}}(u) d u, \quad y>0 .
$$

The density function $p_{*}(x, y)$ of $P_{*}(x, y)$ is given by

$$
p_{*}(x, y)=f_{P_{0}}^{2}(x) e^{-y f_{P_{0}}(x)}, \quad y>0 .
$$

Lemma 1. Let $\xi_{1}, \ldots, \xi_{n}$ be a sequence of i.i.d. random variables with density function $f_{P_{0}}(\cdot)$. Suppose, for a real valued measurable function $h_{n}(\cdot, \cdot)$, that there exists a constant $C$ such that $\sup _{n,(x, y) \in R \times R^{+}}\left|h_{n}(x, y)\right| \leq C$, and suppose that $E\left[h_{n}\left(\xi_{1}, \eta_{1}(n)\right)\right]=0$ for all $n$. Then

$$
\frac{1}{n} \sum_{i=1}^{n} h_{n}\left(\xi_{i}, \eta_{i}(n)\right) \stackrel{a_{\text {a.s. }}}{\rightarrow} 0 \quad \text { as } \quad n \rightarrow \infty .
$$

Proof. The lemma follows by the Borel-Cantelli Lemma and the asymptotic behaviour of the fourth order moments of $n^{-1} \sum h_{n}\left(\xi_{i}, \eta_{i}(n)\right)$. See Ekström (1996) for details.

Lemma 2 Let $\xi_{1}, \ldots, \xi_{n}$ be a sequence of i.i.d. random variables with density function $f_{P_{0}}(\cdot)$. Then for any finite measure $Q$ dominated by $\mu$, under assumptions $A_{2}(i)$ and $A_{2}(i i)$, the random function

$$
V_{\Psi, n}(N, Q)=\frac{1}{n+1} \sum_{i=1}^{n} \max \left(0, \Psi\left(z_{i}(n, Q)\right)-N\right)
$$

converges to zero for all elementary events, uniformly in $n$, as $N \rightarrow \infty$ (i.e. $\left.\sup _{n \geq 1} V_{\Psi, n}(N, Q) \rightarrow 0\right)$. 
Proof. See Lemma 2 in Ranneby and Ekström (1997). In Ranneby and Ekström it is assumed that $Q$ is a probability measure, but it follows easily that the result also holds for the more general case, when $Q$ is a finite measure dominated by $\mu$, as well. (In fact, the result holds for any finite measure $Q$ on the real line.)

Proof of Theorem 1. For the case when $\Psi(t)=\log t$, the lemma follows by the Borel-Cantelli Lemma and the fact that (see Pyke (1965))

$$
\left\{F_{P_{0}}\left(\xi_{(j)}\right)-F_{P_{0}}\left(\xi_{(j-1)}\right): 1 \leq j \leq n+1\right\} \stackrel{D}{=}\left\{Z_{j} / \sum_{i=1}^{n+1} Z_{i}: 1 \leq j \leq n+1\right\},
$$

where $\left\{Z_{j}\right\}_{j=1}^{n+1}$ is an i.i.d. sample from the standard exponential distribution. See Shao and Hahn (1995) for details.

Next, assume that $\Psi(t), t>0$, is bounded from below, i.e. $\inf _{t>0} \Psi(t)>-\infty$. Note that, for $N>0$,

$$
\begin{aligned}
& \frac{1}{n+1} \sum_{j=1}^{n} \min \left(N, \Psi\left(z_{j}\left(n, P_{0}\right)\right)\right)+\frac{\inf _{t>0} \Psi(t)}{n+1} \leq S_{\Psi, n}\left(P_{0}\right) \\
& \leq \frac{1}{n+1} \sum_{j=1}^{n} \min \left(N, \Psi\left(z_{j}\left(n, P_{0}\right)\right)\right)+\frac{\Psi^{+}(n+1)}{n+1}+V_{\Psi, n}\left(N, P_{0}\right),
\end{aligned}
$$

where $V_{\Psi, n}\left(N, P_{0}\right)=(n+1)^{-1} \sum_{i=1}^{n} \max \left(0, \Psi\left(z_{i}\left(n, P_{0}\right)\right)-N\right)$. By Lemma 1 , $\frac{1}{n+1} \sum_{j=1}^{n}\left(\min \left(N, \Psi\left(z_{j}\left(n, P_{0}\right)\right)\right)-E\left[\min \left(N, \Psi\left(z_{j}\left(n, P_{0}\right)\right)\right)\right]\right) \stackrel{\text { a.s. }}{\rightarrow} 0$ as $n \rightarrow \infty$. Furthermore by (3) and a change of variables, with $z=F_{P_{0}}(x)$ and $w=(n+$ 1) $\left(F_{P_{0}}(x+y /(n+1))-F_{P_{0}}(x)\right)$, we have that

$E\left[\min \left(N, \Psi\left(z_{j}\left(n, P_{0}\right)\right)\right)\right]$

$$
\begin{aligned}
= & \int_{-\infty}^{\infty} \int_{0}^{\infty} \min \left(N, \Psi\left((n+1)\left(F_{P_{0}}\left(x+\frac{y}{n+1}\right)-F_{P_{0}}(x)\right)\right)\right) p_{* n}(x, y) d y d x \\
& +\int_{-\infty}^{\infty} \min \left(N, \Psi\left((n+1)\left(1-F_{P_{0}}(x)\right)\right)\right) f_{P_{0}}(x) F_{P_{0}}(x)^{n-1} d x \\
= & \int_{0}^{1} \int_{0}^{(n+1)(1-z)} \min (N, \Psi(w)) \frac{n-1}{n+1}\left(1-\frac{w}{n+1}\right)^{n-2} d w d z \\
& +\int_{0}^{1} \min (N, \Psi((n+1)(1-z))) z^{n-1} d z \\
\rightarrow & \int_{0}^{\infty} \min (N, \Psi(w)) e^{-w} d w \text { as } n \rightarrow \infty .
\end{aligned}
$$

Consequently,

$$
\begin{aligned}
\lim _{n \rightarrow \infty} \frac{1}{n+1} \sum_{j=1}^{n} \min \left(N, \Psi\left(z_{j}\left(n, P_{0}\right)\right)\right) & \stackrel{a . s .}{=} \int_{0}^{\infty} \min (N, \Psi(w)) e^{-w} d w \\
& \rightarrow \int_{0}^{\infty} \Psi(w) e^{-w} d w \text { as } N \rightarrow \infty .
\end{aligned}
$$


This result, together with the inequalities in (4) and Lemma 2, proves Theorem 1. $\mathrm{D}$

Proof of Theorem 2. Let $J_{I}^{\prime}=\left\{j:\left[\xi_{j}, \xi_{j}+\eta_{j}(n) /(n+1)\right] \subseteq I, 1 \leq j \leq n\right\}$ and

$$
\Psi_{M, N}(x)=\left\{\begin{array}{ccc}
-M & \text { if } & \Psi(x) \leq-M \\
\Psi(x) & \text { if } & -M<\Psi(x)<N \\
N & \text { if } & N \leq \Psi(x)
\end{array}\right.
$$

Then

$$
\begin{aligned}
& \varlimsup_{n \rightarrow \infty} \frac{1}{n+1} \sum_{j \in J_{I}} \Psi\left((n+1)\left(F_{Q}\left(\xi_{(j+1)}\right)-F_{Q}\left(\xi_{(j)}\right)\right)\right) \\
& \leq \varlimsup_{n \rightarrow \infty} \frac{1}{n+1} \sum_{j \in J_{I}^{\prime}} \Psi\left(z_{j}(n, Q)\right)+\varlimsup_{n \rightarrow \infty} \frac{\Psi^{+}(n+1)}{n+1} \\
& \leq \varlimsup_{M \rightarrow \infty} \varlimsup_{n \rightarrow \infty} \frac{1}{n+1} \sum_{j \in J_{I}^{\prime}} \Psi_{M, N}\left(z_{j}(n, Q)\right)+\varlimsup_{n \rightarrow \infty} V_{n}(N, Q)
\end{aligned}
$$

An application of Lemma 1 yields

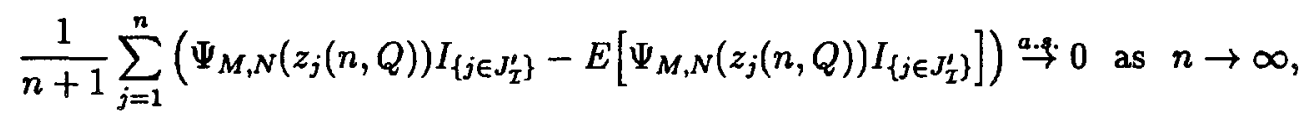
and, by assumption $A_{1}$ it follows as in (5) that, for $j=1, \ldots, n$,

$$
E\left[\Psi_{M, N}\left(z_{j}(n, Q)\right) I_{\left\{j \in J_{\mathcal{I}}^{\prime}\right\}}\right] \rightarrow \int_{\mathcal{I}} \int_{R^{+}} \Psi_{M, N}\left(y f_{Q}(x)\right) p_{*}(x, y) d y d x .
$$

Moreover by Lemma 2 there exists $\varepsilon_{N}$ such that $0 \leq \sup _{n \geq 1} V_{\Psi, n}(N, Q) \leq \varepsilon_{N} \rightarrow 0$ as $N \rightarrow \infty$. Hence almost surely

$$
\begin{aligned}
& \varlimsup_{M \rightarrow \infty} \varlimsup_{n \rightarrow \infty} \frac{1}{n+1} \sum_{j \in J_{\mathcal{I}}^{\prime}} \Psi_{M, N}\left(z_{j}(n, Q)\right)+\varlimsup_{n \rightarrow \infty} V_{n}(N, Q) \\
& \leq \varlimsup_{M \rightarrow \infty} \int_{\mathcal{I}} \int_{R^{+}} \Psi_{M, N}\left(y f_{Q}(x)\right) p_{*}(x, y) d y d x+\varepsilon_{N} \\
& \leq \varlimsup_{M \rightarrow \infty} \int_{I} \int_{R^{+}} \max \left(-M, \Psi\left(y f_{Q}(x)\right)\right) p_{*}(x, y) d y d x+\varepsilon_{N} \\
& \leq \int_{\mathcal{I}} \int_{R^{+}} \Psi\left(y f_{Q}(x)\right) p_{*}(x, y) d y d x+\varepsilon_{N}
\end{aligned}
$$

where the last inequality follows from Fatou's lemma. Letting $N \rightarrow \infty$ we obtain the desired result. 
Proof of Theorem 3. Suppose that $P \neq P_{0}, P \in \overline{\mathcal{P}}$, and, without loss of generality, that $\int_{R} \int_{R^{+}} \Psi\left(y \gamma_{P}(x)\right) p_{*}(x, y) d y d x>-\infty$ holds. Then given $\varepsilon>0$ there is a $\delta(\varepsilon)>0$ such that for every set $\mathcal{A}$, with $P_{0}(\mathcal{A})<\delta(\varepsilon)$, we have $\left|\int_{A} \int_{R^{+}} \Psi\left(y \gamma_{P}(x)\right) p_{*}(x, y) d y d x\right|<\varepsilon$. Moreover by assumption $A_{5}$ (see the proof of Theorem 3.1 in Shao and Hahn (1996)), there exist for each $\delta(\varepsilon)$ a large positive number $M$, a small number $r_{M}>0$ and a finite union of intervals $\mathcal{I}_{M}$ such that $P_{0}\left(\mathcal{I}_{M}\right) \leq \delta(\varepsilon)$ and $g_{P}\left(x, r_{M}\right) \leq M$, for all $x \in \mathcal{I}_{M}^{c}$.

Recall that for each $P \in \mathcal{P}$

$$
S_{\bar{\Psi}, n}(P)=\frac{1}{n+1} \sum_{j=0}^{n} \Psi\left((n+1)\left(F_{P}\left(\xi_{(j+1)}\right)-F_{P}\left(\xi_{(j)}\right)\right)\right) .
$$

Further, define for each $Q \in \overline{\mathcal{P}} \backslash \mathcal{P}$,

$$
S_{\Psi, n}(Q)=\varlimsup_{r \rightarrow \infty} \sup _{P \in b(Q, r) \cap \mathcal{P}} S_{\Psi, n}(P),
$$

where $b(Q, r)=\{P: d(P, Q)<r, P \in \overline{\mathcal{P}}\}$.

The next step is to show, for any fixed $P \neq P_{0}, P \in \overline{\mathcal{P}}$, that there exists a function $T_{P}(M)$ such that

$\varlimsup_{n \rightarrow \infty} \sup _{Q \in b\left(P_{,}, r_{M}\right)} S_{\Psi, n}(Q) \leq T_{P}(M) \rightarrow \int_{R} \int_{R^{+}} \Psi\left(y \gamma_{P}(x)\right) p_{*}(x, y) d y d x \quad$ as $M \rightarrow \infty$.

To do this, $\varlimsup_{n \rightarrow \infty} \sup _{Q \in b\left(P, r_{M}\right)} S_{\Psi, n}(Q)$ will be split into two parts.

Define

$$
J_{M}^{c}=\left\{j:\left[\xi_{(j)}, \xi_{(j+1)}\right] \subseteq \mathcal{I}_{M}^{c}, 0 \leq j \leq n\right\}
$$

and

$$
J_{M}=\{0, \ldots, n\} \backslash J_{M}^{c}
$$

and let $j_{M}$ be the number of elements in $J_{M}$. Then, by the law of large numbers, $j_{M} / n \stackrel{a . s}{\rightarrow} P_{0}\left(\mathcal{I}_{M}\right)$ as $n \rightarrow \infty$. By the concavity of $\Psi$ we get

$$
\begin{aligned}
& \varlimsup_{n \rightarrow \infty} \sup _{Q \in b\left(P, r_{M}\right) \cap \mathcal{P}} \frac{1}{n+1} \sum_{j \in J_{M}} \Psi\left((n+1)\left(F_{Q}\left(\xi_{(j+1)}\right)-F_{Q}\left(\xi_{(j)}\right)\right)\right) \\
& \leq \varlimsup_{n \rightarrow \infty} \frac{1}{n+1} \sum_{j \in J_{M}} \Psi\left(\frac{n+1}{j_{M}}\right) \stackrel{\text { a.s. }}{=} P_{0}\left(\mathcal{I}_{M}\right) \Psi\left(\frac{1}{P_{0}\left(\mathcal{I}_{M}\right)}\right) \rightarrow 0 \text { as } M \rightarrow \infty .
\end{aligned}
$$


Moreover by Theorem 2 the following inequalities hold almost surely,

$$
\begin{aligned}
\varlimsup_{n \rightarrow \infty} & \sup _{Q \in b\left(P, r_{M}\right) \cap \mathcal{P}} \frac{1}{n+1} \sum_{j \in J_{M}^{c}} \log \left((n+1)\left(F_{Q}\left(\xi_{(j+1)}\right)-F_{Q}\left(\xi_{(j)}\right)\right)\right) \\
\leq & \varlimsup_{n \rightarrow \infty} \frac{1}{n+1} \sum_{j \in J_{M}^{c}} \log \left((n+1) \int_{\xi_{(j)}}^{\xi_{(j+1)}} g_{P}\left(x, r_{M}\right) d x\right) \\
\leq & \int_{\mathcal{I}_{M}^{c}} \int_{R^{+}} \Psi\left(y g_{P}\left(x, r_{M}\right)\right) p_{*}(x, y) d y d x \\
\leq & \int_{R} \int_{R^{+}}\left(\Psi\left(y g_{P}\left(x, r_{M}\right)\right) I_{\left\{x \in \mathcal{I}_{M}^{c}\right\}}+\Psi\left(y \gamma_{P}(x)\right) I_{\left\{x \in \mathcal{I}_{M}\right\}}\right) p_{*}(x, y) d y d x \\
& \quad-\int_{R} \int_{R^{+}} \Psi\left(y \gamma_{P}(x)\right) I_{\left\{x \in \mathcal{I}_{M}\right\}} p_{*}(x, y) d y d x .
\end{aligned}
$$

By the monotone convergence theorem, the first double integral on the right hand side above decreases to $\int_{R} \int_{R^{+}} \Psi\left(y \gamma_{P}(x)\right) p_{*}(x, y) d y d x$, and by the dominated convergence theorem the second double integral tends to zero, as $M \rightarrow \infty$. Note that by Jensen's inequality,

$$
\begin{aligned}
\int_{R} \int_{R^{+}} \Psi\left(y \gamma_{P}(x)\right) p_{*}(x, y) d y d x & =\int_{R^{+}} e^{-y}\left\{\int_{R} \Psi\left(\frac{y \gamma_{P}(x)}{f_{P_{0}}(x)}\right) f_{P_{0}}(x) d x\right\} d y \\
& <\int_{R^{+}} \Psi(y) e^{-y} d y \\
& =\int_{R} \int_{R^{+}} \Psi\left(y f_{P_{0}}(x)\right) p_{*}(x, y) d y d x .
\end{aligned}
$$

Define

$$
T_{P}(M)=\int_{\mathcal{I}_{M}^{c}} \int_{R^{+}} \Psi\left(y g_{P}\left(x, r_{M}\right)\right) p_{*}(x, y) d y d x+P_{0}\left(\mathcal{I}_{M}\right) \Psi\left(\frac{1}{P_{0}\left(\mathcal{I}_{M}\right)}\right) .
$$

Now, almost surely,

$\varlimsup_{n \rightarrow \infty} \sup _{Q \in b\left(P, r_{M}\right)} S_{\Psi, n}(Q) \leq T_{P}(M) \rightarrow \int_{R} \int_{R^{+}} \Psi\left(y \gamma_{P}(x)\right) p_{*}(x, y) d y d x \quad$ as $M \rightarrow \infty$, which implies that there exist $\tau_{P}>0$ and $r_{M}>0$ so small such that $\varlimsup_{n \rightarrow \infty} \sup _{Q \in b\left(P, r_{M}\right)} S_{\Psi, n}(Q) \leq \int_{R} \int_{R^{+}} \Psi\left(y \gamma_{P}(x)\right) p_{*}(x, y) d y d x+\tau_{P}<\int_{R^{+}} \Psi(y) e^{-y} d y$.

Next, since for each $\rho>0, \overline{\mathcal{P}} \backslash b\left(P_{0}, \rho\right)$ is compact, there exist finitely many neighbourhoods $b\left(P_{1}, r_{M_{1}}\right), \ldots, b\left(P_{k}, r_{M_{k}}\right)$ which cover $\overline{\mathcal{P}} \backslash b\left(P_{0}, \rho\right)$. Therefore, almost surely,

$$
\begin{aligned}
\varlimsup_{n \rightarrow \infty} \sup _{Q \in \overline{\mathcal{P}} \backslash b\left(P_{0}, \rho\right)} S_{\Psi, n}(Q) & \leq \max _{1 \leq i \leq k}\left(\int_{R} \int_{R^{+}} \Psi\left(y \gamma_{P_{i}}(x)\right) p_{*}(x, y) d y d x+\tau_{P_{i}}\right) \\
& <\int_{R^{+}} \Psi(y) e^{-y} d y
\end{aligned}
$$


Thus by Theorem 1, for any asymptotic MSP estimator $\hat{P}_{\Psi, n}$, the probability that $\sup _{k \geq n} d\left(\hat{P}_{\mathbb{Y}, k}, P_{0}\right)<\rho$ holds, tends to 1 as $n \rightarrow \infty$, as was to be proved.

Proof of Theorem 5. Since all densities $f_{P}, P \in \mathcal{P}$, are unimodal there exist numbers $M_{P}$ such that $f_{P}, P \in \mathcal{P}$, is nondecreasing on $\left(-\infty, M_{P}\right)$ and nonincreasing on $\left(M_{P}, \infty\right)$. Without loss of generality it is assumed that $M_{P_{0}}=0$.

First we show that if the set $\left\{M_{P}\right\}$ is unbounded there exists $K>0$ such that the following inequality holds almost surely,

$$
\varlimsup_{n \rightarrow \infty} \sup _{\left|M_{P}\right|>K} S_{\Psi, n}(P)<\int_{R^{+}} \Psi(y) e^{-y} d y .
$$

For each $\varepsilon>0$ there exists $x_{0}>0$ such that $\delta\left(x_{0}\right)=\int_{-\infty}^{-x_{0}} d F_{P_{0}}(x)+\int_{x_{0}}^{\infty} d F_{P_{0}}(x)<$ $\varepsilon$. Let $C>0$ and put $K=x_{0}(2 C+1)$. If $\left|M_{P}\right|>K$, then $f_{P}(x) \leq 1 /\left(2 x_{0} C\right)$ for $x \in\left[-x_{0}, x_{0}\right]$, and $\int_{-x_{0}}^{x_{0}} f_{P}(x) d x \leq 1 / C$.

Define $J_{K}=\left\{j:\left|\xi_{(j+1)}\right|>x_{0}\right.$ or $\left.\left|\xi_{(j)}\right|>x_{0}, j=0, \ldots, n\right\}, J_{K}^{c}=\{0, \ldots, n\} \backslash J_{K}$, and let $j_{K}$ denote the number of elements in $J_{K}$. Then, by the law of large numbers, $j_{K} / n \stackrel{\text { a.s. }}{\rightarrow} \delta\left(x_{0}\right)$ as $n \rightarrow \infty$. Moreover, by the concavity of $\Psi$,

$$
\begin{aligned}
& \varlimsup_{n \rightarrow \infty} \sup _{\left|M_{P}\right|>K} \frac{1}{n+1} \sum_{j \in J_{K}} \Psi\left((n+1)\left(F_{P}\left(\xi_{(j+1)}\right)-F_{P}\left(\xi_{(j)}\right)\right)\right) \\
& \leq \varlimsup_{n \rightarrow \infty} \frac{1}{n+1} \sum_{j \in J_{K}} \Psi\left(\frac{n+1}{j_{K}}\right) \stackrel{a . s .}{=} \delta\left(x_{0}\right) \Psi\left(\frac{1}{\delta\left(x_{0}\right)}\right)
\end{aligned}
$$

and

$$
\begin{aligned}
& \varlimsup_{n \rightarrow \infty} \sup _{\left|M_{P}\right|>K} \frac{1}{n+1} \sum_{j \in J_{K}^{c}} \Psi\left((n+1)\left(F_{P}\left(\xi_{(j+1)}\right)-F_{P}\left(\xi_{(j)}\right)\right)\right) \\
& \leq \varlimsup_{n \rightarrow \infty} \frac{1}{n+1} \sum_{j \in J_{K}^{c}} \Psi\left(\frac{n+1}{C\left(n+1-j_{K}\right)}\right) \stackrel{\text { a.s. }}{=}\left(1-\delta\left(x_{0}\right)\right) \Psi\left(\frac{1}{C\left(1-\delta\left(x_{0}\right)\right)}\right) .
\end{aligned}
$$

Consequently, almost surely,

$$
\varlimsup_{n \rightarrow \infty} \sup _{\left|M_{P}\right|>K} S_{\Psi, n}(P) \leq \delta\left(x_{0}\right) \Psi\left(\frac{1}{\delta\left(x_{0}\right)}\right)+\left(1-\delta\left(x_{0}\right)\right) \Psi\left(\frac{1}{C\left(1-\delta\left(x_{0}\right)\right)}\right) .
$$

Since $\delta\left(x_{0}\right)<\varepsilon$ it follows, by the assumptions on $\Psi$, that $\lim _{\varepsilon \rightarrow 0} \delta\left(x_{0}\right) \Psi\left(1 / \delta\left(x_{0}\right)\right)=$ 0 and $\lim _{C \rightarrow \infty, e \rightarrow 0} \Psi\left(1 /\left(C\left(1-\delta\left(x_{0}\right)\right)\right)\right)<\int_{R^{+}} \Psi(y) e^{-y} d y$. Thus the right hand side of inequality (7) is less than $\int_{R^{+}} \Psi(y) e^{-y} d y$ for $\varepsilon$ sufficiently small and $C$ sufficiently large. Therefore the inequality (6) holds.

The rest of the proof is identical to the corresponding part of the proof of Theorem 4.1 in Shao and Hahn (1996), where the special case $\Psi(t)=\log t$ is considered. In their proof it is first assumed that $f_{P_{0}}$ has a unique mode $M_{P_{0}}$, 
and use is made of a metric $d$ on unimodal densities, as introduced by Reiss (1973) by means of the Lévy metric for monotone functions. Theorems 2.10, 2.11, 2.12 and 2.17 of Reiss (1973) are also needed. The inequality (6) together with the results of Reiss (1973) justify the consideration of a compact subset of $\overline{\mathcal{P}}$ when $\overline{\mathcal{P}}$ is not compact with respect to $d$. On this compact (sub-) set Shao and Hahn show that assumptions $A_{3}-A_{5}$ are satisfied. The result then easily extends to the case when the mode of $f_{P_{0}}$ is not unique. The $L^{1}$ convergence is a consequence of Theorem 2.17 of Reiss (1973).

\subsection{Proofs of results presented in section 3}

Proof of Theorem 7. The notation of the proof of Theorem 3 will be used.

First, suppose that $P \neq P_{0}, P \in \overline{\mathcal{P}}$, and without loss of generality that $\int_{-\infty}^{\infty} \log \left(\gamma_{P}(x) / f_{P_{0}}(x)\right) d F_{P_{0}}(x)>-\infty$ holds. Define

$$
\begin{aligned}
& K_{M}^{c}=\left\{j:\left[\xi_{(j)}, \xi_{\left(j+m_{n}\right)}\right] \subseteq \mathcal{I}_{M}^{c}, 0 \leq j \leq n-m_{n}+1\right\} \\
& K_{M}=\left\{0, \ldots, n-m_{n}+1\right\} \backslash K_{M}^{c}
\end{aligned}
$$

and let $k_{M}$ be the number of elements in $K_{M}$. Then by Theorem 6 , almost surely,

$$
\begin{aligned}
\varlimsup_{n \rightarrow \infty} & \sup _{Q \in b\left(P, r_{M}\right) \cap \mathcal{P}} \frac{1}{n} \sum_{j \in K_{M}^{c}} \log \left(\frac{F_{Q}\left(\xi_{\left(j+m_{n}\right)}\right)-F_{Q}\left(\xi_{(j)}\right)}{F_{P_{0}}\left(\xi_{\left(j+m_{n}\right)}\right)-F_{P_{0}}\left(\xi_{(j)}\right)}\right) \\
\leq & \varlimsup_{n \rightarrow \infty} \frac{1}{n} \sum_{j \in K_{M}^{c}} \log \left(\frac{\int_{\xi_{(j)}}^{\xi_{\left(j+m_{n}\right)}} g_{P}\left(x, r_{M}\right) d x}{F_{P_{0}}\left(\xi_{\left(j+m_{n}\right)}\right)-F_{P_{0}}\left(\xi_{(j)}\right)}\right) \\
\leq & \int_{T_{M}^{c}} \log \left(\frac{g_{P}\left(x, r_{M}\right)}{f_{P_{0}}(x)}\right) d F_{P_{0}}(x) .
\end{aligned}
$$

Moreover there exists a positive constant $C$ such that, almost surely,

$$
\begin{aligned}
\varlimsup_{n \rightarrow \infty} & \sup _{Q \in b\left(P_{,} r_{M}\right) \cap \mathcal{P}} \frac{1}{n} \sum_{j \in K_{M}} \log \left(\frac{F_{Q}\left(\xi_{\left(j+m_{n}\right)}\right)-F_{Q}\left(\xi_{(j)}\right)}{F_{P_{0}}\left(\xi_{\left(j+m_{n}\right)}\right)-F_{P_{0}}\left(\xi_{(j)}\right)}\right) \\
& \leq \varlimsup_{n \rightarrow \infty} \frac{1}{n} \sum_{j \in K_{M}} \log \left(\frac{m_{n} / k_{M}}{F_{P_{0}}\left(\xi_{\left(j+m_{n}\right)}\right)-F_{P_{0}}\left(\xi_{(j)}\right)}\right) \\
& \leq-P_{0}\left(\mathcal{I}_{M}\right) \log P_{0}\left(\mathcal{I}_{M}\right)+C \sqrt{P_{0}\left(\mathcal{I}_{M}\right)}
\end{aligned}
$$

where the last inequality is shown in the proof of Theorem 3 in Ekström (1997a). Define, for each $P \in \mathcal{P}, R_{n}^{\left(m_{n}\right)}(P)=S_{n}^{\left(m_{n}\right)}(P)-S_{n}^{\left(m_{n}\right)}\left(P_{0}\right)$, and for each $Q \in \overline{\mathcal{P}} \backslash \mathcal{P}, R_{n}^{\left(m_{n}\right)}(Q)=\varlimsup_{\lim _{r \rightarrow 0}} \sup _{P \in b(Q, r) \cap \mathcal{P}} R_{n}^{\left(m_{n}\right)}(P)$. Then by $(8)-(9)$ and by 
the monotone and dominated convergence theorems,

$$
\begin{aligned}
& \varlimsup_{n \rightarrow \infty} \sup _{Q \in b\left(P_{\left.r_{M}\right)}\right.} R_{n}^{\left(m_{n}\right)}(Q) \\
& \quad \leq \int_{\mathcal{I}_{M}^{c}} \log \left(\frac{g_{P}\left(x, r_{M}\right)}{f_{P_{0}}(x)}\right) d F_{P_{0}}(x)-P_{0}\left(\mathcal{I}_{M}\right) \log P_{0}\left(\mathcal{I}_{M}\right)+C \sqrt{P_{0}\left(\mathcal{I}_{M}\right)} \\
& \quad \rightarrow \int_{R} \log \left(\frac{\gamma_{P}(x)}{f_{P_{0}}(x)}\right) d F_{P_{0}}(x)<0 \text { as } M \rightarrow \infty,
\end{aligned}
$$

where the last inequality follows from Jensen's inequality. Consistency can be established as in the proof of Theorem 3.

\section{References}

[1] Bahadur, R.R. (1971). Some Limit Theorems in Statistics. SIAM, Philadelphia.

[2] Bauer, H. (1972). Probability Theory and Elements of Measure Theory. Holt, Rinehart and Winston.

[3] Cheng, R.C.H. and Amin, N.A.K. (1983). Estimating parameters in continuous univariate distributions with a shifted origin. J. R. Statist. Soc. B, 45, 394-403.

[4] Csiszár, I. (1963). Eine Informationstheoretische Ungleichung und ihre Anwendung auf den Beweiz der Ergodizität von Markoffschen Ketten. Magyar Tud. Akad. Mat. Kutató Int. Közl., 8, 85-108.

[5] Ekström, M. (1994). On the consistency of the maximum spacing method. Statistical Research Report 1994-11, University of Umeå.

[6] Ekström, M. (1996). Strong consistency of the maximum spacing estimate. Theory Probab. Math. Statist., 55.

[7] Ekström, M. (1997a). Strong limit theorems for sums of logarithms of $m$ th order spacings. Research Report No. 7. Dept. of Mathematical Statistics, Umeå University.

[8] Ekström, M. (1997b). Generalized maximum spacing estimators. Research Report No. 6. Dept. of Mathematical Statistics, Umeå University.

[9] Ghosh, K. and Jammalamadaka, S.R. (1996). A general estimation method using spacings. In preprint.

[10] Pyke, R. (1965). Spacings. J. R. Statist. Soc. B, 27, 395-449. 
[11] Ranneby, B. (1984). The maximum spacing method. An estimation method related to the maximum likelihood method. Scand. J. Statist., 11, 93-112.

[12] Ranneby, B. and Ekström, M. (1997). Maximum spacing estimates based on different metrics. Research Report No. 5. Dept. of Mathematical Statistics, Umeå University.

[13] Reiss, R.D. (1973). On measurability and consistency of maximum likelihood estimates for unimodal densities. Ann. Statist., 1, 888-901.

[14] Roeder, K. (1990). Density estimation with confidence sets exemplified by superclusters and voids in the alaxies. J. Amer. Statist. Assc., 85, 617-624.

[15] Shao, Y. and Hahn, M.G. (1995). Limit theorems for the logarithm of sample spacings. Statist. Probab. Lett., 24, 121-132.

[16] Shao, Y. and Hahn, M.G. (1996). Strong consistency of the maximum product of spacings estimates with applications in nonparametrics and in estimation of unimodal densities. In preprint.

[17] Wald, A. (1949). Note on the consistency of the maximum likelihood estimate. Ann. Math. Statist., 20, 595-601. 
